\title{
Le rapport à l'expertise scientifique d'enseignants de sciences du secondaire du Gabon face à une polémique environnementale
}

\section{Christophe Ndong Angoué}

\section{(Q) OpenEdition}

Édition électronique

URL : http://journals.openedition.org/ere/825

DOI : 10.4000/ere.825

ISSN : 2561-2271

Éditeur

Centr'ERE

Référence électronique

Christophe Ndong Angoué, « Le rapport à l'expertise scientifique d'enseignants de sciences du secondaire du Gabon face à une polémique environnementale », Éducation relative à l'environnement [En ligne], Volume 11 | 2014, mis en ligne le 20 décembre 2013, consulté le 21 février 2020. URL : http://journals.openedition.org/ere/825; DOI : 10.4000/ere.825 


\title{
Le rapport à l'expertise scientifique d'enseignants de sciences du secondaire du Gabon face à une polémique environnementale
}

\author{
Christophe Ndong Angoué
}

Le concept de "questions socialement vives » (QSV) est apparu récemment dans le monde éducatif francophone (Legardez et Simonneaux, 2006) dans le prolongement de certains travaux anglo-saxons ${ }^{1}$ portant sur les «socioscientific issues» ou « controversial socioscientific issues » (Cardoso, Bride et Thénard-Duvivier, 2008; Albe, 2009). Ces questions sont globalement perçues dans l'espace éducatif francophone comme des questions complexes porteuses de discussions, de désaccords, d'incertitudes et de controverses à travers lesquelles les réponses ne sont pas simples, uniques ou universelles et mobilisent les représentations, les valeurs, les intérêts des acteurs sociaux (Legardez et Simonneaux, 2006; Albe, 2009; Legardez et Simonneaux, 2011). Elles sont vivaces dans la société, car elles suscitent des débats entre différents acteurs sociaux, mobilisent des expertises scientifiques souvent contradictoires et les gouvernants doivent se prononcer à leurs sujets (Albe, 2010-2011). Leur vivacité peut varier selon les époques et les circonstances (Legardez et Simonneaux, 2011). En outre, ce sont des questions qui sont prégnantes dans l'environnement social et médiatique des acteurs éducatifs qui en ont connaissance même de manière sommaire (Albe, 2009). Leur intégration dans le champ scolaire reposerait sur la volonté d'outiller les futurs citoyens face aux incertitudes de la « science en train de se faire» (Albe, 2009). Ce qui leur permettrait de mieux comprendre les enjeux contemporains portant sur l'usage de l'expertise scientifique dans nos sociétés dites démocratiques. Dans le contexte éducatif gabonais et dans la perspective d'une éducation plus citoyenne des élèves, des problématiques sociales ${ }^{2}$ ont été intégrées dans le programme gabonais de sciences de la vie et de la terre (SVT) du secondaire depuis l'année 1997 à travers le programme d'éducation en matière de population (EMP). Pour ce qui a trait particulièrement à l'enseignement des questions environnementales, ce programme EMP permettrait par 
exemple l'enseignement du thème portant sur l'exploitation des ressources naturelles dans la perspective du développement durable et viserait une bonne compréhension des interactions entre populations-environnement-développement (Ministère de l'Éducation nationale et coll., 2000). Cependant, enseigner une telle question dans une perspective d'éducation citoyenne et dans le contexte du cours de sciences au secondaire peut s'avérer périlleux pour les enseignants de sciences gabonais. C'est à ce titre qu'il nous a paru intéressant de cerner la manière dont des enseignants de sciences au Gabon assumaient de telles problématiques afin de mieux comprendre leurs prises positions face à un tel enseignement.

2 Une revue de littérature sur la manière dont les enseignants de sciences s'approprient et traitent de ces questions dans différents contextes nous a conduit à identifier plusieurs perspectives théoriques ${ }^{3}$ sur le sujet. Autour des postures épistémologiques que les enseignants adoptent dans un tel contexte, des auteurs tels que Albe (2009) et Aikenhead (2006) vont respectivement identifier la tendance des enseignants à accorder une grande légitimité aux discours experts notamment lorsqu'ils doivent caractériser l'origine des controverses et la manière dont s'élaborent les connaissances en sciences, et le fait que tout en étant favorables à l'enseignement des sciences dans la perspective humaniste ${ }^{4}$, ces derniers semblaient se voir comme les dépositaires d'une certaine vision empirico-réaliste ${ }^{5} \mathrm{du}$ monde après leur formation universitaire. Ces études vont ainsi mettre l'emphase sur des considérations d'ordre disciplinaire et épistémologique pour caractériser la manière dont des enseignants s'approprient et traitent des QSVE. En nous appuyant sur de telles considérations, on peut alors se poser la question suivante: Quel rapport à l'expertise scientifique les enseignantes et enseignants de sciences du secondaire du Gabon développent-ils au regard de leurs postures épistémologiques lorsqu'ils doivent se prononcer sur une question socialement vive environnementale?

\section{Le rapport au savoir : un outil théorique pour cerner l'épistémo-logie enseignante}

3 Pour arriver à cerner le rapport entretenu par les enseignants avec la question de l'expertise scientifique et donc l'épistémologie qui en découle, le cadre théorique du rapport au savoir tel qu'il est développé par Bernard Charlot (1997) nous a semblé le plus pertinent. Dans le champ de la didactique des sciences, Pouliot (2007, p. 52) en remettant en forme la définition de Bernard Charlot sur la question définit le rapport aux experts scientifiques (et donc à l'expertise scientifique) comme : «[...] un ensemble de relations que des personnes entretiennent avec des activités, des relations interpersonnelles, des lieux, des personnes, des situations, des obligations, etc. [...] ». Ce rapport peut être associé à des dimensions épistémique (rapport au monde), sociale (rapport aux autres) et identitaire (rapport à soi-même). Cet outil théorique nous a amenés à comprendre notamment à travers la dimension épistémique ou épistémologique que nous présentons dans cet article, l'expérience singulière des enseignants de sciences du Gabon aux prises avec une question socialement vive environnementale (QSVE) locale dont les dimensions scientifiques sont issues de la « science en train de se faire » et qui interpellent leur rapport à l'expertise scientifique. 


\section{L'expertise scientifique dans la QSVE}

\section{Regard sur la notion d'expertise scientifique}

4 Si l'on se réfère à l'éclairage théorique de Roqueplo (1997), le mot «expert » peut recouvrir une double signification selon le contexte dans lequel il est utilisé. Dans un contexte de négociation politique dans lequel l'expert scientifique représenterait un pays, ce mot aurait le sens de compétence ou de qualification, car l'expert est utilisé dans ce cas comme une personne suffisamment qualifiée "pour intervenir avec compétence dans le processus de décision auquel il doit participer » (Roqueplo, 1997, p. 12). Ici, l'expert jouerait alors un rôle de négociateur politique pour défendre les intérêts d'un État. Dans un autre contexte, qui fonde par ailleurs la théorisation de cet auteur, le mot expert peut désigner une personne « dont la fonction est de formuler une expertise » c'est-à-dire une connaissance capable d'apporter un éclairage scientifique pertinent et exhaustif sur une situation problématique donnée. Dans cette perspective, le rôle de l'expert n'est pas de décider, mais de produire de la connaissance pour une prise de décision éclairée. Nous notons cependant à la suite d'Albe (2009, p. 46) qu'en acceptant de fournir cette connaissance à ceux qui le sollicitent, l'expert scientifique s'inscrit inéluctablement dans "une situation paradoxale ». Soumis à des probléma-tiques complexes, il se place en position de "transgression de son savoir " (Roqueplo, 1997, p. 45) par le fait que «ses croyances, ses convictions globales, son idéologie, ses solidarités, ses préjugés, sa classe sociale, son appartenance sociale, etc. » sont mis en perspective de manière consciente ou non bien que moulés dans le langage qu'il connaît le mieux c'est-à-dire celui de la science. C'est à ce titre que Roqueplo ${ }^{6}$ propose une institutionnalisation des procédures de préparation permanente d'expertise dont la responsabilité reviendrait aux scientifiques pour répondre aux caractères pluridisciplinaires des problèmes auxquels sont confrontés les experts. En considérant ce caractère quelque peu subjectif de l'expertise scientifique, des auteurs comme Ancori (2009) vont alors proposer de recourir à une définition plus nuancée de l'expert en le présentant comme « un sujet supposé savoir et dire le vrai à propos d'une question précise s'inscrivant dans le domaine particulier de compétence dont il est crédité, et dont le dire fournit un socle à la prise de décision parmi d'autres également possibles dans un contexte correspondant à un autre domaine de compétence ». Ce regard permettrait d'envisager l'expertise scientifique d'une part à la croisée d'autres formes d'expertise (Larochelle et Désautels ; 2006) mais aussi offrirait l'éventualité de son usage critique et démocratique par les acteurs éducatifs (Albe, 2009) notamment dans le contexte d'un enseignement citoyen des QSVE.

\section{L'expertise scientifique entre " science en train de se faire " et "science faite »}

Plusieurs positionnements épistémologiques ${ }^{7}$ peuvent être identifiés à travers la littérature sur la manière dont on pourrait envisager la production des savoirs scientifiques et l'activité scientifique. Nous tenons cependant à souligner ici la nécessité d'inscrire l'expertise scientifique dans la dialectique entre la "science en train de se faire " et la "science faite " afin de jeter un regard plus fécond sur la conception des sciences mobilisée par les enseignants lorsqu'il s'agit pour eux de mettre en lumière les processus d'expertise en jeu dans une QSVE. L'expression 
«science en train de se faire » prend son ancrage dans une certaine idée de la socialité des sciences et de l'activité scientifique émanant de travaux en éducation aux sciences (Albe, 2009, Richard et Bader, 2010), mais aussi de travaux en sociologie des sciences (Callon, Lascoumes et Barthes, 2001; Latour, 2001; Ancori, 2009). Latour (2001) la présente comme une science incertaine, risquée qui a des liaisons nombreuses avec la politique, la société et dans laquelle les faits sont construits et évaluer comme des valeurs. Albe (2009) et Ancori (2009) la voient comme une science qui n'est pas neutre, car ses capacités d'investigation sont assujetties à des choix politiques, économiques et sociaux chargés de valeurs. Cette conception des sciences lorsqu'elle est mobilisée pour analyser les processus d'expertise suggérerait ainsi que les propositions scientifiques sur une controverse socioscientifique soient considérées comme conditionnelles et non prescriptives et que les résultats fournis par les experts subissent une traduction (Callon, Lascoumes et Barthes, 2001) dans le monde réel notamment lorsqu'il s'agit d'en évaluer les risques (Ancori, 2009). Cette vision de l'activité scientifique contrasterait ainsi avec celle qui est dite de la "science faite » (Latour, 2001) à saveur plus empiricoréaliste qui envisagerait plutôt l'activité scientifique de manière neutre et fondée sur un ensemble de certitudes, de faits, de données dites objectives (Albe, 2009). La mobilisation d'une telle conception des sciences aboutirait par conséquent à un traitement purement scientifique d'une controverse socioscientifique (Ancori, 2009) en occultant d'autres formes de considérations en jeu dans un tel contexte.

\section{La pertinence du recours à une cartographie des controverses}

6 Pour Bruno Latour (2011, en ligne) :

Cartographier une controverse, c'est [...] lister les positions en présence en décrivant pour chacune d'elles par qui elles sont portées, des scientifiques, des industriels, des organisations non gouvernementales [...] avec quels arguments. Le but ultime est d'aider le citoyen à se forger une opinion sur ces questions controversées.

7 Elle a donc comme principale vertu de rendre visible et lisible une problématique complexe en permettant une représentation des acteurs impliqués et des positions débattues. La cartographie permet alors de présenter la " science en train de se faire ${ }^{8}$ en soulignant l'interpénétration de la science, de la politique, de l'économie sur une question (Albe, 2008). Dans notre étude nous l'avons utilisée comme un outil méthodologique afin d'amener des enseignants de sciences du secondaire du Gabon à mieux expliciter leurs propos sur les processus d'expertise en jeu dans la QSVE, c'est-àdire à mieux identifier les acteurs impliqués, leurs arguments, sur quoi portent leurs désaccords et à se prononcer sur la question.

\section{Le cas particulier des risques environnementaux et de santé publi- que de l'exploitation de l'uranium à Mounana}

Ce cas met en exergue des polémiques entre experts scientifiques autour de la dangerosité radioactive du site d'exploitation de cette ressource bien que ce site ait été réaménagé par l'entreprise d'exploitation afin de freiner ses émanations radioactives. Ces polémiques trouvent leur essence dans les plaintes de populations faisant état dans 
la localité de Mounana au Sud Est du Gabon de nombreux décès d'anciens mineurs par des cancers, et ce, malgré le réaménagement du site. Les populations seraient alors sujettes à de multiples pathologies pulmonaires, gynécologiques et dermatologiques 9 . L'entreprise d'exploitation est accusée d'avoir volontairement caché des informations sur les risques que les travailleurs prenaient en étant si proches du minerai radioactif sans masques, sans gants, sans informations et sans formation sur la dangerosité de leur profession. Ce déficit d'informations (savamment orchestré aux dires de l'élu local de l'ancienne ville minière lors d'un point de presse durant l'année $2008^{10}$ ) aurait de graves conséquences considérant certaines pratiques des populations consistant par exemple à tremper du manioc ${ }^{11}$ dans les cours d'eau irradiés.

9 Face aux plaintes des populations, les experts mandatés par l'opérateur économique (Areva) et par le gouvernement gabonais notamment l'agence internationale de l'énergie atomique (AIEA) estimèrent respectivement qu'il n'y avait pas de preuves scientifiques directes de l'incidence des activités d'exploitation de l'uranium sur la santé des populations et que le réaménagement du site était conforme aux standards internationaux ${ }^{12}$. C'est à ce titre que les organisations non gouvernementales (ONG) nationale (Brainforest) et internationale (Sherpa ${ }^{13}$, Médecins du monde et la CIIRAD ${ }^{14}$ ) ont diligenté une contre-expertise scientifique sur la question et montrèrent que dans cette ville plus de deux millions de tonnes de résidus radioactifs furent déversées dans les rivières entre 1961 et 1975 et que de tels résidus posaient des problèmes radiologiques à long terme. La procédure de réaménagement du site d'exploitation semble ainsi à leurs yeux, très insuffisante, car ce réaménagement a consisté à recouvrir certains secteurs radioactifs de remblais en terre végétale alors que les déchets radioactifs dont la période physique est très longue sont toujours dans le sol. Il apparaît alors plausible ${ }^{15}$ que cette radioactivité puisse avoir un impact sur les eaux et l'air et donc sur les populations de cette localité.

\section{Les différents enjeux entourant l'exploitation de l'uranium au Gabon}

Cartographier une telle question permet d'en identifier les divers acteurs, leur gamme de positionnements, sur quoi portent leurs désaccords, mais aussi les enjeux scientifiques et sociopolitiques parcourant les différentes positions. L'enjeu scientifique porterait notamment sur les critères scientifiques définissant avec certitude la dangerosité radioactive du site même après son réaménagement et sur la notion de " preuve scientifique » évoquée par les experts de l'opérateur économique. En effet, les experts de l'AIEA vont fonder leurs arguments sur le respect des standards internationaux alors que ceux de la CIIRAD vont plutôt faire référence à des éléments de temporalité autour de la période radioactive de l'uranium et sur la durée de l'exploitation pour réfuter ces arguments. Les experts de l'opérateur économique par contre évoqueraient la notion de "preuve scientifique directe» de l'impact des activités d'extraction sur l'environnement et la santé des populations pour relativiser la responsabilité de l'opérateur économique sur les décès et les pathologies constatées dans la région et contrer ainsi les appréhensions de la population sur l'impact de la radioactivité sur la ville de Mounana. Ce positionnement des experts de l'opérateur économique semble révéler implicitement certains intérêts particuliers. L'enjeu sociopolitique serait par contre lié à l'absence coupable d'informations sur les risques d'exploitation de l'uranium, métal stratégiquement important dans le développement de l'énergie nucléaire. Alors que les divers acteurs sociaux impliqués dans la question 
aspirent à un accès à l'information pour une participation efficiente aux débats sur les mesures à prendre pour sécuriser les populations touchées, l'opacité informationnelle constatée et le recours exclusif à l'expertise scientifique pour produire de l'information sur la question semblent témoigner d'une forme de "déficit démocratique " et constituent de ce fait un problème sociopolitique majeur.

\section{Quelques éléments méthodologiques et analytiques}

11 Pour la production des données, l'entretien semi-dirigé a été la technique utilisée pour accéder aux idées des sujets sur l'expertise scientifique. Cette technique qualitative consiste selon Savoie-Zajc $(2009$, p. 340) « [...] a une interaction verbale animée de façon souple par le chercheur [...]. Grâce à cette interaction, une compréhension riche du phénomène à l'étude sera construite conjointement avec l'interviewé ». Nous l'avons utilisé pour construire une compréhension socialement située de notre objet à travers les significations que les enseignants lui attribuent. Onze enseignants de sciences de la vie et de la terre ont été recrutés dans les lycées de Libreville au Gabon. Leurs âges variaient entre 35 et 50 ans et leur ancienneté dans l'enseignement allait de 7 à 24 ans. Avec chaque enseignant, nous avons réalisé deux entretiens individuels durant lesquels nous avons traité successivement de l'expertise scientifique de manière générale dans l'entretien 1 , puis de manière spécifique dans le deuxième entretien en nous appuyant sur la cartographie de la controverse environnementale. Le but étant de permettre aux enseignants dans un premier temps de définir ce qu'est un expert, de souligner leurs connaissances de départ de la controverse et des acteurs impliqués, des arguments évoqués et des qualités que les experts doivent posséder pour se prononcer sur la question étudiée. Dans un deuxième temps, ils devaient réagir sur la cartographie, identifier les acteurs impliqués et leurs arguments, porter un regard critique sur les désaccords entre experts et les positions qu'ils soutiennent.

Une analyse de contenu thématique a été menée (Paillé et Muchielli, 2008). Elle s'est effectuée en deux étapes. La première a consisté à identifier à travers le corpus collecté et retranscrit mot à mot, les idées centrales énoncées au regard de nos préoccupations de recherche afin d'en identifier les différents thèmes ${ }^{16}$. Puis, ces thèmes une fois identifiés ont été regroupés en fonction de leur complémentarité sous des thèmes plus généraux. La deuxième étape, plus interprétative, a permis d'établir des liens entre ces différents thèmes généraux et nos préoccupations de recherche.

\section{Premiers résultats}

13 Le premier constat que nous faisons en analysant les verbatim du premier entretien est le manque d'informations qu'ont les enseignants interrogés sur les experts impliqués sur la controverse en question. Certes, la majorité d'entre eux ont entendu parler de l'impact environnemental de l'exploitation à travers certains reportages faisant état du mécontentement des populations. Cependant, ces enseignants n'avaient pas la mesure des enjeux socioscientifiques autour de la question. À cet effet, la cartographie lors du deuxième entretien a été un outil très important, car elle leur a permis de mieux identifier les acteurs impliqués, leurs arguments et donc de mieux prendre position sur la question. Les propos du participant 1 (P1) en témoignent : 
En fait, là-dedans [dans la cartographie] je suis plus informé que ce que je savais. On m'avait déjà dit qu'il $\mathrm{y}$ avait des problèmes là-bas avec la radioactivité due à l'exploitation de ce minerai, ça, je le savais. Je savais que [...] y a la santé de la population qui risque d'être ébranlée par cette exploitation. Ça, je le savais. Mais je ne savais pas que c'était arrivé jusqu'à ce point, jusqu'à la mort de certains travailleurs dans les sites. Donc, je suis beaucoup plus imprégné et beaucoup plus surpris de ce qui est là-dedans [la cartographie] et je crois que ça s'accorde bien avec ce que j'avais déjà appris sur [...] au niveau des différentes informations que j'ai pu recueillir ça et là (P1).

14 Autour de l'expertise scientifique, les constats que nous faisons vont s'articuler autour de cinq thèmes à savoir la caractérisation de l'expertise scientifique, le regard porté sur les désaccords entre experts, le regard critique porté sur la notion de «standards internationaux », la crédibilité accordée en fonction de l'organisme de l'expert et l'influence de considérations sociales sur les positions d'experts.

\section{Caractérisation de l'expertise scientifique}

\section{Des conceptions diverses de l'expertise scientifique}

Nos résultats préliminaires illustrent des conceptions diverses de l'expertise scientifique à travers lesquelles l'expert est vu comme un chercheur ou une personne de terrain qui trouve des solutions à des problèmes spécifiques (P1, P3, P5, P8). Celui-ci donne un éclairage pertinent (P11) sur une situation qui échappe à la majorité des individus (P5). Pour ce faire, il doit avoir beaucoup de connaissances et une maîtrise de son domaine (P2, P3, P4, P6, P9, P10, P11). Il doit également avoir une expérience de laboratoire (P1, P3, P4, P7, P8) et de terrain (P7, P8). C'est un guide ou un conseiller (P1, $\mathrm{P} 2)$, qui doit dire la réalité (P2) et tirer la sonnette d'alarme (P4) afin de permettre une prise de décision éclairée sur une situation problématique (P2, P7, P9, P10). Quelques propos significatifs :

P1 : Un expert scientifique est celui qui est à même de trouver [...] des solutions relatives [...] à son domaine de sciences... est celui qui est à même de résoudre toutes les interrogations que l'on se pose dans les sciences dans son domaine précis $[\ldots]$.

P2 : [...] Leur rôle [les experts scientifiques] c'est de donner la bonne information parce que ce n'est pas eux qui décident. Le décideur est le politique, mais l'expert scientifique doit être à mesure de dire au décideur voilà [...]. Donc c'est un conseil, c'est un guide. [...]

P4 : Son rôle, je pense serait de tirer la sonnette d'alarme sur tout ce qui se passe dans le monde, la gestion du monde, la gestion durable, gestion de l'environnement, le développement durable avec les travaux qu'ils font, les données qu'ils ont. Il serait là pour attirer l'attention des uns et des autres sur les dérives qu'il y a.

\section{Une expertise fondée sur certaines qualités}

16 Pour les différents participants lors du premier entretien, les experts scientifiques impliqués dans la controverse doivent être des professionnels de l'uranium (P.3) avoir une bonne connaissance scientifique de l'uranium (P.1, P.2); avoir du courage (P 2 ; P.5) et une force de caractère pour préserver leurs crédibilités (P 6). Ils doivent être capables de restituer les faits tels qu'ils les ont observés (P.11). En outre, ces derniers doivent utiliser une méthode d'investigation transparente pour permettre a fortiori une contre-expertise (P.7 ; P.11). Ils doivent faire preuve de valeurs morales et éthiques 
(P.4; P.8; P.9 ; P 10). Ces experts devraient également regarder les faits froidement avec objectivité lors de leurs investigations (P.5).

\section{Regard sur les désaccords entre experts}

\section{Difficultés des enseignants à considérer qu'il y ait désaccords dans une science «normale »}

Confrontés à la cartographie, certains enseignants ont éprouvé un malaise en constatant qu'il existait des désaccords entre les différents experts scientifiques impliqués. P1 va par exemple trouver que le système (la cartographie) est défectueux, car présentant des points de vue divergents entre experts. À son avis pour qu'une réponse soit trouvée, il faut que tous les experts aient un même regard sur la situation.

P1: Bien. Ici j'ai en face de moi un système qui est...défectueux. Pourquoi parce que... nous avons plusieurs organismes qui...contiennent des experts qui donnent des informations divergentes. [...]. Il faut revoir ce système de fonctionnement pour le rendre de plus en plus efficace pour que les informations qui remontent vers le gouvernement soient uniques. Ils font le même travail tous ces experts, mais ils donnent des informations divergentes [...].

18 Les participants P1, P2, P3 et P6 considèrent normal quant à eux que les experts ne soient pas d'accord, car ils ont des intérêts différents, mais souhaitent néanmoins qu'un moyen soit trouvé pour les mettre d'accord et qu'une solution soit trouvée au problème. Pour trancher cette question, plusieurs (P1, P2, P8, P11) estiment que le gouvernement a un rôle déterminant à jouer dans la prise de décision, car c'est ce dernier qui est le garant du bien-être des populations. Plusieurs participants pensent en outre qu'il est nécessaire que d'autres experts plus indépendants donnent leurs avis sur la question pour qu'une solution soit trouvée (P1, P2, P3, P10, P11). Concernant toujours les désaccords entre experts scientifiques, trois enseignants interrogés pensent que si les différents experts avaient les mêmes paramètres d'appréciation du problème, ils verraient la même chose ( $\mathrm{P} 4, \mathrm{P} 5, \mathrm{P} 6)$.

\section{Un regard critique porté sur la notion de « standards internationaux »}

19 Plusieurs ont questionné la notion de « respect des standards internationaux » (P1, P6, P3) mise en exergue par les experts de l'AIEA militant notamment pour que de tels standards soient définis en commun accord par les différents protagonistes.

P1: Pourquoi est-ce que nous ne sommes pas suffisamment informés? Parce que j'ai l'impression que la population gabonaise dans la plus grande dans de euh le plus grand nombre n'est pas suffisamment informée. Quels sont les standards internationaux, d'abord? Qu'appelle-t-on standards internationaux? Et par rapport à ces standards internationaux, qu'est-ce qui est fait sur le terrain ? Et par rapport à ce qui est fait sur le terrain, comment est-ce que ces standards internationaux sont gérés au quotidien sur le terrain ? Il y a pas mal d'informations que nous ne connaissons pas qui nous échappent...

P6: Si les experts de l'AIEA soutiennent que les aménagements ont été faits selon les standards internationaux, ça revient à remettre sur la table la question des standards. Qu'est-ce qu'on entend par les standards internationaux ? Est-ce que ce qui se passe en Europe est exactement ce qui se passe au Gabon ou bien en Afrique du Sud ou bien en Chine? [...]. 
P3 : [...] quand ils disent [que] le réaménagement a été effectué selon les standards internationaux, je pense qu'à un moment les experts de l'AIEA devraient dépasser les standards internationaux [...] est-ce que vraiment la radioactivité a été vraiment condensée. Il faut qu'il ait des mesures de vérification parce que la nature peut dépasser les normes internationales.

\section{Crédibilité accordée en fonction de l'organisme de l'expert (entreprise privée, AIEA, ONG)}

20 Autour de la crédibilité accordée aux experts scientifiques dans la QSVE, un seul participant (P.4) semble accorder du crédit aux experts scientifiques de l'agence internationale de l'énergie atomique (AIEA), car pour lui cette agence ne peut être partisane dans cette affaire du fait de son profil international. La très grande majorité des participants accordent plus de crédit aux experts des ONG, car leurs positions semblent plus proches des préoccupations des populations sur la question. Le degré de crédibilité accordé aux experts rattachés à l'entreprise privée est par ailleurs presque nul. Un participant ne s'est pas prononcé sur la crédibilité des experts.

\section{L'influence des considérations sociales sur les positions d'experts}

21 Autour de la cartographie, les participants ont reconnu presque tous de manière explicite que les experts scientifiques pouvaient être influencés du fait de la corruption lorsqu'ils sont invités à se prononcer sur la question controversée de l'exploitation de l'uranium au Gabon. En effet, pour eux s'il y a désaccords sur des paramètres scientifiques appréciés par les différents experts, cela est révélateur d'un problème d'éthique. D'où la nécessité selon eux que l'on fasse intervenir une expertise plus indépendante dans la QSVE. Les propos suivants tentent de l'illustrer :

P1: Moi je pense qu'ils [les experts scientifiques] sont influençables de par la corruption.

P4 : Tout à fait [les positions des experts peuvent être influencées] puisqu'on a des experts scientifiques [...] qui ne donnent pas forcément les mêmes résultats pour des données scientifiques qui normalement devraient être les mêmes partout. Donc comment pour les paramètres qu'on mesure qu'on apprécie et pour lesquels les normes devraient être les mêmes, on a des avis contraires ? Ça veut dire qu'il y a un problème qui se pose par rapport à l'appréciation même de la chose et par rapport à l'éthique, voilà.

\section{Discussion et conclusion}

L'étude que nous menons tente d'offrir des pistes intéressantes pour comprendre la posture épistémologique des enseignants de sciences et leur rapport à l'expertise scientifique dans un contexte d'analyse d'une QSVE. Les premiers résultats auxquels nous sommes parvenus montrent que pour les enseignants interrogés, dans les circonstances «normales" c'est-à-dire sans polémique, les experts scientifiques seraient des personnes, dont les qualités morales, éthiques, l'objectivité et le bagage intellectuel devraient permettre d'apporter des solutions pertinentes à des problèmes qui se posent dans la société. Par conséquent, l'émergence de "désaccords " entre experts scientifiques dans un contexte de polémiques semble créer en eux un malaise. Ces désaccords ne cadreraient pas avec leurs conceptions du fonctionnement normal de 
l'expertise scientifique, car selon eux, si les différents experts avaient les mêmes critères d'appréciation, ils aboutiraient aux mêmes résultats sur l'incidence de l'exploitation de l'uranium sur l'environnement et la santé des populations. Cette vision des processus d'expertise semble alors reposer sur une conception des sciences à saveur empirico-réaliste. Or selon notre revue des écrits, des considérations de nature un peu plus subjective peuvent être envisagées dans les processus d'expertise notamment dans la perspective de la "science en train de se faire». Nous notons par ailleurs que les enseignants vont avoir un rapport à l'expertise qui sera fonction de l'affiliation de ces derniers à un organisme particulier. Signalons pour finir que les enseignants font preuve d'une grande réflexivité en étant capables d'identifier certains enjeux liés aux processus d'expertise lorsqu'ils questionnent notamment la notion de «standards internationaux » évoquée par les experts de l'AIEA.

\section{BIBLIOGRAPHIE}

Aikenhead, G.S. (2006). Science Education for everyday life : evidence-based practice. New York and London : Teachers College press.

Albe, V. (2010-2011). Changements climatiques à l'école : Pour une éducation sociopolitique aux sciences et à l'environnement. Éducation relative à l'environnement : Regards-Recherches-Réflexions, 9, 95-114.

Albe, V. (2009). Enseigner les controverses. Rennes : Presses Universitaires de Rennes.

Albe, V. (2008). Culture scientifique et technique « scientific and technological literacy»: Quelles visées éducatives? Quelles références pour les savoirs scolaires ? Dans Gardiès, C., Fabre, I., Duchamp, C. et Albe, V. (dir.), Éducation à l'information et éducation aux sciences : quelles formes scolaires? Rencontres Toulouse EducAgro, 26 et 27 mai 2008, ENFA. Toulouse : Cépaduès-éditions.

Albe, V. et Simonneaux, L. (2002). L'enseignement des questions scientifiques socialement vives dans l'enseignement agricole : Quelles sont les intentions des enseignants ? Aster 34, 131-136.

Ancori, B. (2009). Expertise et citoyenneté : Les Grecs anciens et nous. De l'Agora antique aux forums hybrides modernes. Revue d'anthropologie des connaissances, 3, 485-529.

Callon, M ; Lascoumes, P. et Barthe, Y. (2001). Agir dans un monde incertain. Essai sur la démocratie technique. Paris : Seuil.

Cardoso, A ; Bride, P et Thénard-Duvivier, F. (2008). L'enseignement des questions socialement vives : objet d'étude et sujet d'actualité ? Dans Thénard-Duvivier, F. (dir.), L'enseignement des questions socialement vives en histoire et géographie. Actes du colloque, 14-15 mars 2008. Paris : SNES /CVUH.

Cavet, A. (2007). L'enseignement des « questions vives » lien vivant, lien vital, entre école et société. La lettre d'information de la VST, 27. Récupéré de : ife.ens-lyon.fr/vst/DA-Veille/27mai-2007.pdf 
Charlot, B. (1997). Du rapport au savoir. Éléments pour une théorie. Paris : Anthropos. Girault, Y. et Lhoste, Y. (2010). Opinions et savoirs : positionnements épistémologiques et questions didactiques. Revue RDST, 1, 29-66.

Kelly, T. (1986). Discussing Controversial Issues : "Four Perspectives on the Teacher's Role". Theory and Research in Social Education, 14, 113-138.

Larochelle, M. et Désautels, J. (2006). L'éducation aux sciences et le croisement des expertises. Dans Legardez, A. et Simonneaux, L. (dir), L'école à l'épreuve de l'actualité. Enseigner les questions vives (p. 61-77). Paris : ESF.

Latour, B. (2001). Le métier de chercheur regard d'un anthropologue ( $2^{\mathrm{e}}$ édition revue et corrigée). Paris : INRA Éditions.

Latour, B. (2011). Nous construisons des outils pour évaluer les controverses. La recherche, 456, p. 76. Récupéré le 28 février 2013 sur le site de la revue : www.larecherche.fr/idees/entretien-dumois/nous-construisons-outils-evaluer-controverses-01-10-2011-72624

Legardez, A. et Simonneaux, L. (2006). L'école à l'épreuve de l'actualité. Paris : ESF. Legardez, A. et Simonneaux, L. (2011). Développement durable et autres questions d'actualité : les questions socialement vives dans l'enseignement et la formation. Dijon : Educagri éditions. Legendre, R. (2005). Le Dictionnaire actuel de l'éducation, $3^{\mathrm{e}}$ édition. Montréal : Guérin. Ministère de l'Éducation nationale ; Institut Pédagogique national et Programme IEC multisectorielle. (2000). Curriculum de BiologieGéologie Collège. UNESCO/FNUAP. Paillé, P. et Mucchielli, A. (2008). L'analyse qualitative en sciences humaines et sociales ( 2 e édition). Paris : Armand Colin.

Pouliot, C. (2007). Appréhension estudiantine d'une controverse sociotechnique et rapport aux experts scientifiques: Une étude de cas. Thèse de doctorat inédite. Université Laval.

Reis, P. et Galvão, C ; Teaching controversial Socio-Scientific Issues in Biology and Geology classes : A case study. Electronic Journal of Science Education, 13(1) 2009. ejse.southwestern.edu/ volumes/v13n1/articles/art7-reis.pdf (Page consultée le 15 avril 2010).

Richard, V. et Bader, B. (2010). Re-presenting the social construction of science in light of the propositions of Bruno Latour : For a renewal of school conception of science in secondary schools. Science Education, 94(4), 743-749.

Roqueplo, P. (1997). Entre savoir et décision, l'expertise scientifique. Paris : INRA Éditions. Sadler, T.D., Amirshokoohi, A., Kazempour, M. et Allspaw, K.M. (2006). Socioscience and Ethics in science classrooms : Teacher Perspectives and strategies. Journal of Research in Science Teaching, 43, 353-376.

Savoie-Zajc, L. (2009). L'entrevue semi-dirigée. Dans Gauthier, B. (dir.), Recherche sociale - De la problématique à la collecte de données (p. 337-360). Sainte-Foy : Presses de l'Université du Québec.

Simonneaux, L. (2003). Les savoirs « chauds » entre science et valeurs. Dans Astolfi, J.P. (dir.), Éducation et formation. Nouvelles questions, nouveaux métiers (p. 181-202). Paris : ESF.

Simonneaux, L. (2010). Introduction des questions scientifiques socialement vives dans l'enseignement agricole français : Implication des recherches en didactique dans la formation des enseignants. Dans Hasni, A. et Lebeaume, J. (dir.), Enjeux contemporains de l'éducation scientifique et technologique (p.81-123). Ottawa : Les Presses de l’Université d'Ottawa. 


\section{NOTES}

1. Dans le monde de la recherche anglophone, les « socioscientific issues » sont vues notamment d'une part comme des questions ayant un intérêt social et impliquant une dimension scientifique (Driver, Leach, Millar et Scott, 1996 in Albe, 2009, p. 27) ou comme des dilemmes sociaux impliquant la science ou la technologie (Sadler, 2004 in Albe 2009, p.27); d'autre part comme des dilemmes moraux à travers lesquels diverses valeurs sont sollicitées. Dans la situation didactique, il s'agira alors d'identifier, de clarifier ces valeurs tout en développant l'attitude critique des élèves (Albe, 2009).

2. Dans le contexte éducatif gabonais, bien que l'enseignement des QSV ne soit pas une piste adoptée actuellement dans les programmes d'enseignement, les problématiques qui se rapprochent de cet enseignement peuvent être identifiées dans le programme d'éducation en matière de population (EMP).

3. Certains auteurs (Sadler, Amirshokoohi, Kazempour et Allspaw, 2006) vont mettre l'accent sur la place que les enseignants accordent à l'éthique et aux valeurs dans les socioscientific issues (SSI). D'autres vont envisager les QSV sous l'angle des représentations sociales (Simonneaux, 2003) ou des intentions des enseignants (Albe et Simonneaux, 2002). Autour des stratégies éducatives (discussion ou situations-débats) mises en œuvre dans un tel contexte, certains chercheurs (Kelly, 1986; Reis et Galvão, 2006) vont non seulement identifier les différentes postures épistémologiques que les enseignants adoptent, mais aussi les différentes contraintes qu'ils vivent dans l'utilisation de ces situations éducatives.

4. Pour Aikenhead (2006), la perspective humaniste est une idéologie qui milite non seulement pour le traitement des questions sociales controversées dans le cours de sciences, mais également pour la présentation de la science comme une entreprise humaine inscrite dans un milieu social et conduite par différentes communautés sociales et scientifiques.

5. L'empirico-réalisme est une vision du monde à la fois empiriste et réaliste mettant de l'avant une connaissance scientifique reflet d'une réalité objective indépendante du sujet connaissant (réalisme) et s'élaborant à partir d'observation de faits et de données sensorielles suscitant une hypothèse que l'expérience viendrait mettre à l'épreuve (empirisme) (Legendre, 2005).

6. C'est sur ce point d'ailleurs que nous nous démarquons quelque peu des propositions théoriques de Roqueplo, car pour nous, bien qu'en soulignant le caractère quelque peu subjectif des processus d'expertise scientifique, il semble réifier le monopole de l'expertise détenue par les scientifiques au détriment d'autres formes d'expertises souvent en jeu dans les controverses socioscientifiques.

7. L'article de Girault et Lhoste (2010) présente autour de la dialectique opinions et savoirs, différents positionnements épistémologiques s'articulant successivement autour de l'épistémologie rationaliste s'inspirant essentiellement des travaux de Bachelard et de Canguilhem; de l'épistémologie s'appuyant sur les «sciences studies" qui se placent en opposition à la précédente posture épistémologique et qui militent pour une production située, intersubjective et négociée des connaissances scientifiques; et autour d'une épistémologie réhabilitant les savoirs populaires (Traditional Ecological Knowledge) ou les savoirs écologiques traditionnels (Indigenous Knowledge).

8. La manière dont se présentent l'activité des scientifiques et la fabrication des savoirs dans les controverses socioscientifiques nous autorise à les désigner par l'assertion «science en train de se faire ".

9. Ces constats ont été publiés dans un rapport en avril 2007 par des organisations non gouvernementales. CRIIAD. (2007). Les conditions d'exploitation de l'uranium par les filiales d'Areva... et les normes ISO. mondialisation.ca/index.php?context= viewArticle\&code=CRI20070424\&articleId=5476 (consulté le 12 octobre 2010). 
10. Moutsinga, C. (2008). Gabon: La société française Areva empoisonne à l'uranium les populations gabonaises. www.radiocemac.com/index.php/Societe-/Societe/Gabon-La-societefrancaise-Avera-empoisonne-a-luranium-des-populations-gabonaises.html (consulté le 20 octobre 2010).

11. Aliment de base des populations locales.

12. Source: [En ligne] nucleaire-nonmerci.net/actualite/areva-contamination-uranium.html (consulté le 23 octobre 2010).

13. Sherpa est un groupe de juristes qui défend les droits des travailleurs et des populations de toutes nationalités face aux multinationales. [En ligne] www.asso-sherpa.org/association/notremission

14. C'est la Commission de Recherche et d'Information Indépendantes sur la Radioactivité.

15. Moutsinga, C. (2008). Gabon: La société française Areva empoisonne à l'uranium les populations gabonaises. www.radiocemac.com/index.php/Societe-/Societe/Gabon-La-societefrancaise-Avera-empoisonne-a-luranium-des-populations-gabonaises.html (Consulté le 20 octobre 2010).

16. Le thème est défini par Paillé et Mucchielli (2008, p.170) comme "un ensemble de mouvements permettant de cerner ce qui est abordé dans l'extrait du corpus». Il donne des indications sur la teneur des propos et n'a pas une valeur interprétative immédiate.

\section{RÉSUMÉS}

L'émergence du champ éducatif des questions socialement vives (QSV) permet désormais l'étude en contexte scolaire de problématiques sociales en lien avec l'environnement et la santé. L'analyse de ces problématiques socioscientifiques environnementales controversées invite à un usage raisonné et critique de l'expertise scientifique en contexte scolaire. Cet article vise à présenter quelques résultats préliminaires de notre étude doctorale dans laquelle nous examinons la manière dont des enseignants de sciences du secondaire au Gabon envisagent la question de l'expertise scientifique face à une polémique environnementale locale mettant en scène des experts d'horizons divers. Nous précisons leur posture épistémologique et leur rapport à l'expertise lorsqu'ils sont placés en situation d'analyse d'une cartographie présentant cette polémique. Les résultats illustrent que leur rapport à l'expertise scientifique lorsqu'il est contextualisé en fonction d'une polémique précise tend à valoriser la position de certains experts plutôt que d'autres, tout en mobilisant une conception des sciences à saveur empirico-réaliste.

Socially controversial issues is an emerging field in education. It allows the study, within school context, of social issues related to environment and health. The analysis of these controversial socioscientific environmental issues invites to a rational and critical use of scientific expertise in school context. This article presents some preliminary results of a doctoral research which examines how Gabon high school science teachers consider scientific expertise regarding a local environmental controversy involving experts from diverse backgrounds. We identify their epistemological position and their relationship to expertise when they are placed in a situation to analyze a mapping of this controversy. Results show that their relationship to scientific expertise, when contextualized with regards to a specific controversy, tends to value some experts position more than others, all the while mobilizing a view of science that is more empirical-realistic. 


\section{AUTEUR}

\section{CHRISTOPHE NDONG ANGOUÉ}

Christophe Ndong Angoué est enseignant de sciences de la vie et de la terre au secondaire au Gabon. Il a obtenu une maîtrise en didactique des sciences à l'Université Laval à Québec en 2005. Il s'intéresse à l'épistémologie scolaire et à l'enseignement des questions socialement vives environnementales dans le cours de sciences. Il poursuit actuellement des études de doctorat en didactique sur ces questions à l'Université Laval. 